\title{
Sul significato della presenza di metano nelle manifestazioni esalative e idrotermali
}

\author{
ALFONSO DI NOI
}

\section{1. - Premessa.}

Già da alcuni anni F. Penta ( ${ }^{1}$ ) ha posto il problema del significato genetico da attribure agli idrocarburi riscontrati in talune manifestazioni esalative ed idrotermali naturali.

Sull'argomento lo scrivente ha condotto uno studio, del quale col presente articolo dà in sintesi i risultati.

iे da notare, anzitutto, che lo studio in parola de stato condotto soltanto su basi rhimico-fisiche. Ciò comporta implicitamente la necessità di un controllo su altre basi.

Sono stati esaminati alcuni equilibri schematici, allo scopo di studiarne l'andamento in condizioni di pressione e temperatura paragonabili a quelle che si presume carattelizzino le masse ignee più o meno profonde. E implicita, in questa fase dello studio,l ipotesi semplilicativa che i prodotti gassosi in seno ai fusi, o rifusi, siano chimicamente inerti rispetto alla massa silication.

Per quanto riguarda l'approssimazione dei calcoli si è ritenuto di non introdurre le correzioni per la "fugacità ", conformemente a quanto fatto da $\mathbf{A}$. J. Ellis (-) in un recentissimo lavoro.

Ciò perchè il carattero puramente indicativo dell'indagine non giustifica la maggiore laboriosità dei calcoli che i roefficienti di fugacita comporterebbero. Basta pensare che i calcoli sono riferiti a miseugli ipotetici, aventi funzione di "modello " dei fenomeni studiati, per i quali è stata assunta una composizione iniziale, la composizione, cioè, relativa a condizioni normali di pressione e temperatura, che, a rigore, è arbitraria.
D'altro canto, è anche da osservare che in roncomitanza con le pressioni maggiori, sono in genere da attendersi anche temperature più elevate, per cui è presumibile che i coefficienti di fugaciti non siano tanto discosti dall'unità da togliere ai risultati il loro valore, sia pule soltanto indicativo.

Per quanto liguarda poi l'aspetto cimetico dol problema, esso non ha importanza che in casi particolari, data la scala geologicar rlei tompi che, in generale, interessano.

Sono da attendersi eccezioni solo nei processi rulcanici particolarmente veloci; non tanto nei fenomeni eflusivi, poichè le clevate temperature della lava rendono elevate anche le velocita di reazione, quanto nei fenomeni esplosivi, che sono accompagnati da grandi e repentine variazioni di pressione e temperatura.

Circa il campo delle temperature che interessano, un primo elemento di riferimento ì formito dalle misure relative alle lave fuse.

Se la letteratura è sufficiente a fornire elementi circa le temperature delle lave effondenti ed appare legittima l'estrapolazione per i magmi estremamente superficiali, non altrettanto può dirsi per quelli profondi. Comunque come valore massimo assumeremo quello di 1300 oC che $\mathrm{A}$. Rittmamn ( $\left.{ }^{3}\right)$ attribuisce al magma subcrostale.

Molto più bassse sarebbero, viceversal, le temperature relative alle masse plutoniche propriamente dette. Bowen, ritato da Wahlstrom ('), ad esempio, osserva (he la temperatura di granitizzazione in particolari contizioni può variare nell'intervallo 300650 oC; d'alt to canto, Rittmamm segnala una temperatura massima di 800 oc. 
Per quanto riguarda, poi, le pressioni ̀̀ apparso opportuno limitare il rampo ai valori compresi tra quelli relativi alla libera atmosfera e alla profondità dell'ordine di grandezza della diecina di migliaia di metri, ossia, in cifre tonde, tra 1 e 3000 atmosfere. d pressione atmosferica la dissociazione è molto spinta per temperature di $1000^{\circ} \mathrm{K}$, pari a 727 od; nelle dette condizioni la frazione molare di equilibrio non raggiunge il valore di 0,14 ; per temper"ature superiori ai $1400^{\circ} \mathrm{K}$, colrispondenti a 1127 oC, la dissociazione è praticamente completa.

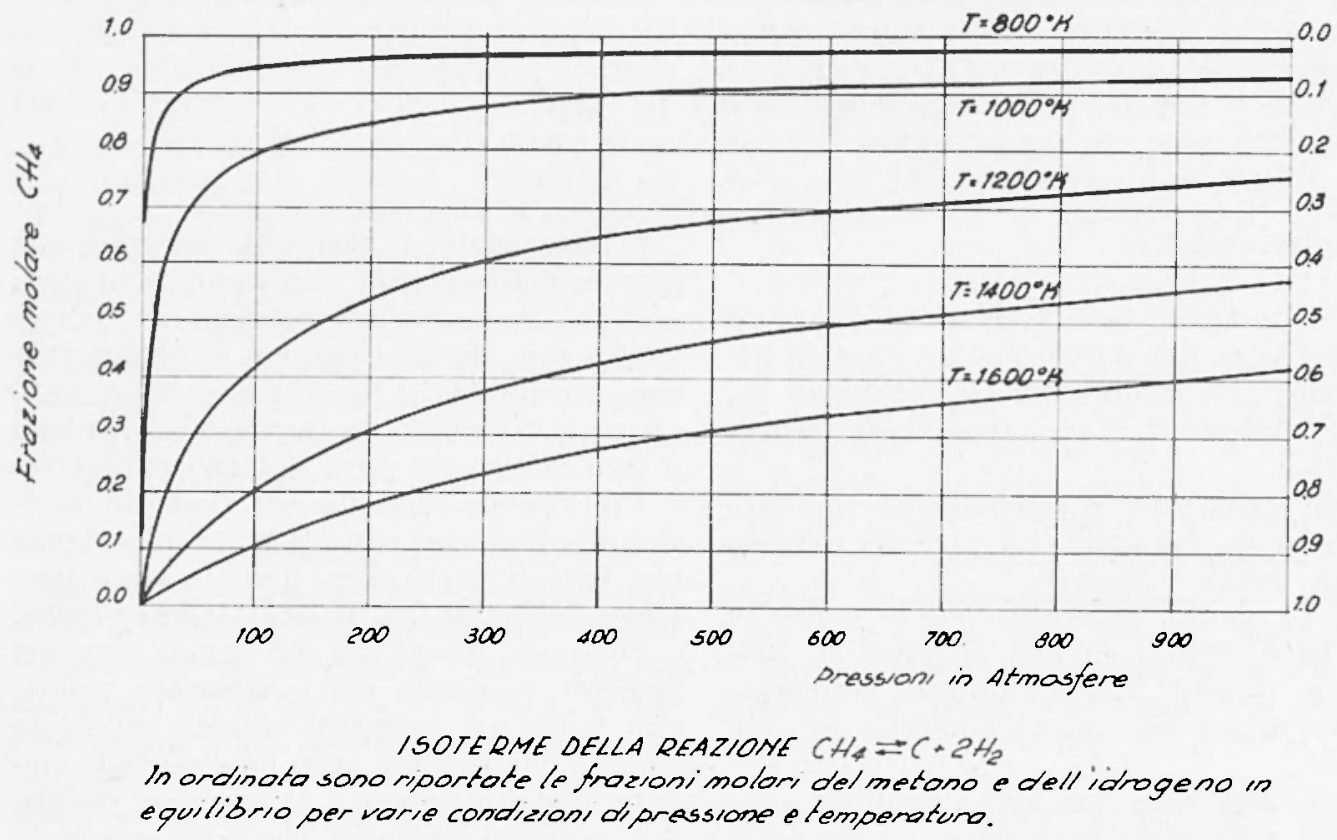

Fig. 1

\section{2. - Pirolisi del Metixo.}

Ta prima reazione schematica studiata è quella di pirolisi del metano secondo la

$$
\mathrm{CH}_{1} \longleftrightarrow \mathrm{C}+2 \mathrm{H}_{2}
$$

Ie costanti di equilibrio relative alle varie temperature sono state riprese da G. Egloff (5); i risultati dei calcoli sono mppresentati in lig. 1.

Ia pirolisi del metano è favorita dalle alte temperature e dalle basse pressioni.

Per quanto riguarda le condizioni di temperatura, la dissociazione non si verifica praticamente più al disotto dei $600^{\circ} \mathrm{K}$, pari a $327^{\circ} \mathrm{C}$. A questo valore, infatti, l'entità della frazione molare di metano in equilibrio è maggione di 0,94 alla pressione atmosferica, ed è prossima all'unità a 20 atmosfere.
Bisogna ancora aggiungere, però, che l'equilibrio alle alte temperature, è sensibilmente influenzato dalle pressioni, per cui la frazione molare, almeno nel campo di temperature che ci interessa, è sempre superiore a 0,3 se la pressione supera le 500 atm.

\section{3. - REAZIONE FRA METANO E ALTRI CON- PONENTI DEI GAS MAGMATIGI.}

Isar decomposizione del metano può aver luogo anche secondo le seguenti leazioni

$$
\begin{aligned}
& \mathrm{CH}_{4}+2 \mathrm{H}_{2} \mathrm{O}_{\text {vap }} \longrightarrow \mathrm{CO}_{2}+4 \mathrm{H}_{2} \quad \text { [1] } \\
& \mathrm{CH}_{4}+\mathrm{H}_{2} \mathrm{O}_{\mathrm{vap}} \longleftrightarrow \mathrm{CO}+3 \mathrm{H}_{\mathrm{r}} \quad \text { [2] } \\
& \mathrm{CH}_{1}+\mathrm{CO}_{2} \quad \longleftrightarrow 2 \mathrm{CO}_{-} 2 \mathrm{H}_{2} \quad \text { [3] }
\end{aligned}
$$

Di queste è stato studiato l'andamento, nell'ipotesi che i sistemi iniziali siano mi- 
scugli stechiometrici dei composti che figurano al secondo membro. Si è potuto accertare che l'andamento quantitativo delle Jeazioni non è sostanzialmente diverso, aImeno dal punto di vista che qui interessa.

Poichè i gas magmatici sono formati in prevalenza da vapor d'acqua, e ciò sposta sensibilmente l'equilibrio delle reazioni [1] e [2] nel senso della decomposizione del metano, lo studio della [1] è stato ulteriormente approfondito; è stato, cioè, esaminato il comportamento di una miscela di $\mathrm{H}_{2} \mathrm{O}, \mathrm{CO}_{2}$ e $\mathrm{H}_{2}$ avente la seguente composizione iniziale (espressa mediante le frazioni molari)

$$
n_{H_{2} \mathrm{O}}=0,89, n_{\mathrm{Co}_{2}}=0,10, n_{\mathrm{H}_{2}}=0,01 .
$$

Nei calcoli sono stati introdotti i valori delle costanti di equilibrio riportati da A. J. Illis, nel campo di temperatura fra 400 e $1000^{\circ} K$, mentre per i valori $1200^{\circ}$ e $1600^{\circ} K$ si è proceduto al calcolo mediante le formule approssimate di Ulich.

I risultati sono riportati in tabella 1 e rappresentati nelle fig. 2 e 3 .

E da avvertire, ancora, che la frazione molare del metano è stata convenzionalmente ritenuta trascurabile per valori inferiore a $10^{-7}$.

Il calcolo dimostra che, per temperature inferiori a $500^{\circ} \mathrm{K}$ (corrispondenti a $227^{\circ} \mathrm{C}$ ) se la pressione supera qualche centinaio di atmosfere, il metano è praticamente indecomposto; inoltre, anche per basse pressioni, la frazione molare del metano inde- composto è superiore al $50 \%$ del massimo valore possibile in relazione alla composizione iniziale; ciò corrisponde a un valore di $n_{\mathrm{CH}_{4}}$ sempre superiore a 0,0015 .

Per temperature comprese tra i $500^{\circ}$ e i $700^{\circ} \mathrm{K}$, ossia per l'intervallo $227^{\circ} \div 427^{\circ} \mathrm{C}$, la frazione molare del $\mathrm{CH}_{4}$ is ancora notevole, salvo che per pressioni di poche atmosfere.

Per temperature superiori ai $1000^{\circ} K$ la frazione molare del $\mathrm{CH}_{4}$ diminuisce sensibilmente, in particolare a $1600^{\circ} K$, corrispondenti a $1327^{\circ} \mathrm{C}$ il metano praticamente scompare fintanto che la pressione non supera le 500 atmosfere. Per pressioni superiori a questo valore permane sempre una certa frazione indissociata, tanto più grancle quanto più elevata è la pressione.

4. - INTERFERENZA DI ALTRE REAZIONI. REAZIONE DEL GAS D'ACQUA E DISSOCIAZIONE DEL VAPOR D'ACQUA.

Volendo studiare un modello di ulteriore approssimazione, è stata presa in esame una reazione, per cosi dire secondaria, la quale, inserendosi nell'andamento della precedente ne influenza l'equilibrio.

Come reazione secondaria è stata considerata la così detta reazione del gas l'acqua. E stato cioè, preso in esame il sistema di equilibrio

$$
\begin{aligned}
& \mathrm{CO}_{2}+4 \mathrm{H}_{2} \longleftrightarrow \mathrm{CH}_{4}+2 \mathrm{H}_{2} \mathrm{O} \\
& \mathrm{CO}_{2}+\mathrm{H}_{2} \longleftrightarrow \mathrm{CO}+\mathrm{H}_{2} \mathrm{O}
\end{aligned}
$$

\section{Tabella 1}

Reazione: $\mathrm{CO}_{2}+4 \mathrm{II}_{2} \longleftrightarrow \mathrm{CH}_{4}+2 \mathrm{H}_{2} \mathrm{O}$

Frazioni molari di $C H_{4}$ in equilibrio per varie condizioni di pressione e temperatura

I.a composizione iniziale ̀̀: $n_{u_{n}}=0,89 ; n_{\mathrm{CO}_{2}}=0,10 ; n_{H_{2}}-0,01$

\begin{tabular}{llcccccr}
\hline$P^{\prime}$ (Atmosfere) & 1 & 100 & 500 & 800 & 1000 & 2000 & 3000 \\
\hline$T=500^{\circ} \mathrm{K}$ & 0,0016 & 0,0024 & $\sim 0,0025$ & $\sim 0,0025$ & $\sim 0,0025$ & $-0,0025$ & $\sim 0,0025$ \\
$T=600^{\circ} \mathrm{K}$ & 0,0001 & 0,0019 & 0,0022 & 0,0023 & 0,0023 & 0,0023 & 0,0024 \\
$T=700^{\circ} \mathrm{K}$ & trasc. & 0,0009 & 0,0017 & 0,0018 & 0,0019 & 0,0020 & 0,0021 \\
$T=1000^{\circ} \mathrm{K}$ & trasc. & $3.10^{-6}$ & $1.10^{-5}$ & $2.10^{-5}$ & $3.10^{-5}$ & $1.10^{-5}$ & $2.10^{-4}$ \\
$T=1200^{\circ} \mathrm{K}$ & trasc. & trasc. & $1.10^{-6}$ & $4.10^{-6}$ & $8.10^{-6}$ & $3.10^{-5}$ & $6.10^{-5}$ \\
$T=1600^{\circ} \mathrm{K}$ & trasc. & trasc. & trasc. & $2.10^{-7}$ & $3.10^{-7}$ & $1.10^{-6}$ & $2.10^{-6}$ \\
\hline
\end{tabular}




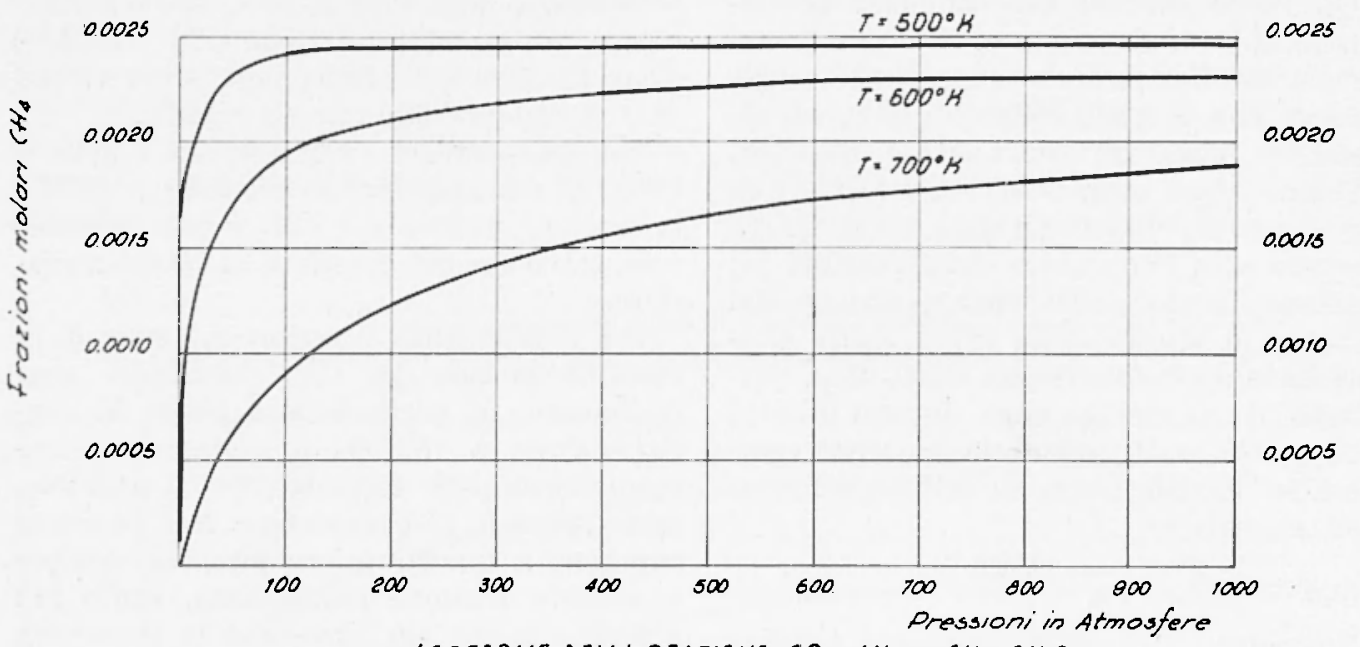

ISOTERME DELLA REAZIONE $\mathrm{CO}_{2}+4 \mathrm{H}_{2}=\mathrm{CH}_{4}+2 \mathrm{H}_{2} \mathrm{O}$

In ordinata sono mportate le frazioni molori di CHA in eovilibrio ser varie condizioni di pressio. ne e temperatura; il sistema ba composicione iniziale: $n_{H_{2}} \mathrm{O}-0.89 ; \mathrm{CO}_{3} .010 ; n_{\mathrm{H}_{1}}-0.01$.

Fig. 2

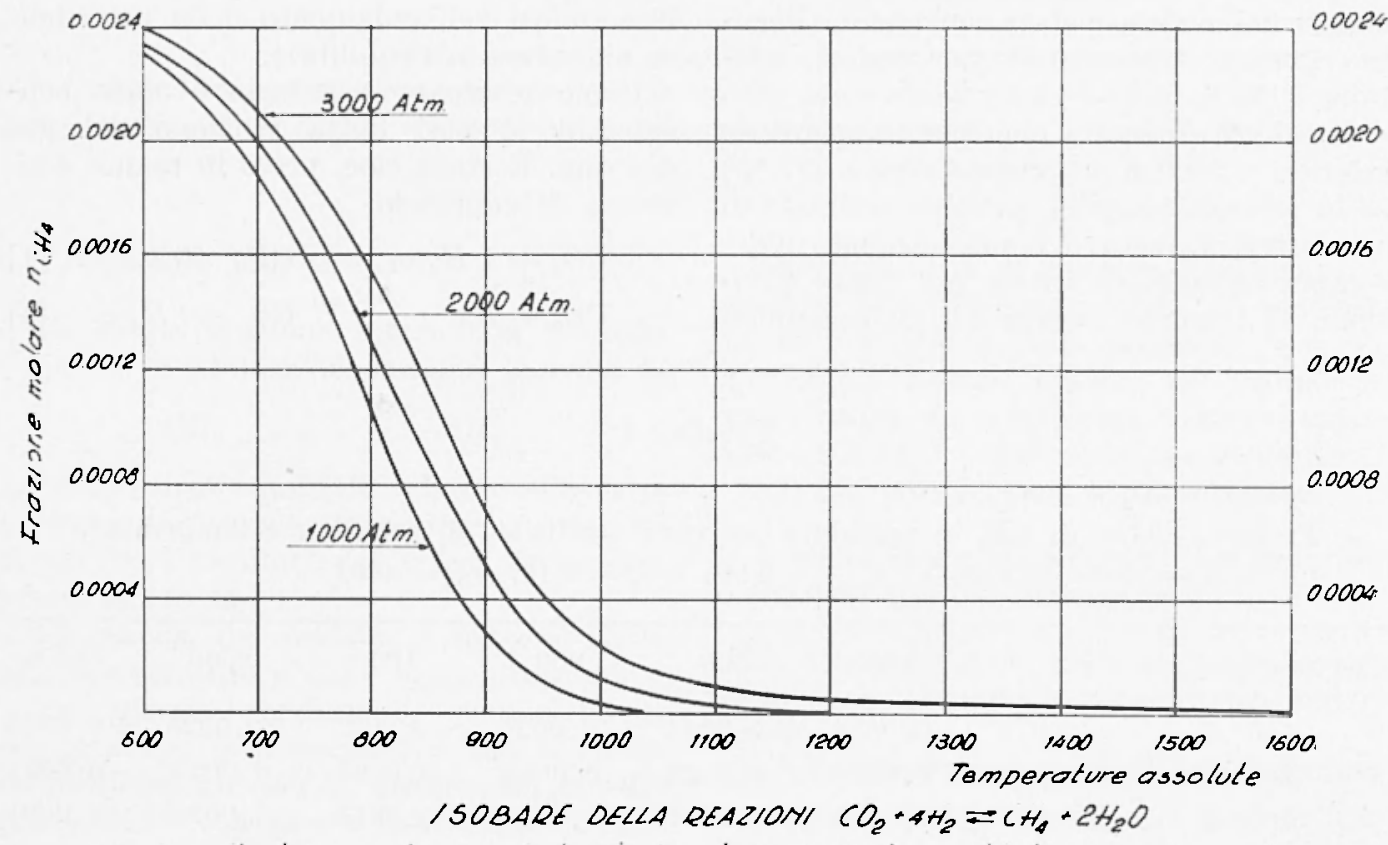

In ordinato sono riportate le frazioni molari di Cits in equilibrio per varie condi. ziani di equilibrio; it sustema ha camposizime iniziale $n_{H_{0} 0}=0.89:{ }^{n} \mathrm{CO}_{2}=0.10: n_{4_{3}}-0.01$

Fig. 3 
I valori delle costanti d'equilibrio per la reazione [t] sono stati desunti da J. Eggert $\left(^{6}\right)$.

L'effetto della reazione [4] è ovviamente quello di sottrarre $\mathrm{CO}_{2}$ alla reazione [1], ostacolando in tal modo la formazione del $\mathrm{CH}_{4}$. La tonalità termica fa prevedere questa azione tanto più intensa quanto più elevata è la temperatura; d'altro canto tale azione è indipendente dalla pressione, per(hè la [4] decorre senza variazione di volume.

Ora noi abbiamo visto quale sia il comportamento della [1] alle alte temperature, e, in particolare, come il $\mathrm{CH}_{1}$ sia instabile a $1600^{\circ} \mathrm{K}$ sotto pressioni relativamente basse. Nel delineato campo di pressione e tempelature la reazione del gas d'acqua non avrà altro risultato notevole che far comparire nel miscuglio gassoso il $\mathrm{CO}$. Pin interessante appare, quindi, l'effetto della reazione [4], nei riguardi della stabilità del metano, sotto forti pressioni.

Alle basse temperature, già salppiamo come la formazione del $C_{4}$ sia favorita, ma non saluanno da prevedere sensibili interferenze da parte della reazione del vapor d'acqua, poichè essa è ostacolata in quelle condizioni di temperature. Per queste ragioni si è ritenuto opportuno limitare lo studio dell'influenza della formazione del gas d'acqua alle alte temperature e alle alte pressioni.

I risultati dei calcoli, sempre nell'ipotesi che la composizione iniziale sia quella gia detta, sono riportati in tabella 2 .
Dal confronto dei dati riportati in tabella 2 con quelli di tabella 1 segue che la reazione del gas dacqua amplia verso più alte pressioni il campo di instabilità del metano pur senza valiare sostanzialmente il quadro generale precedentemente delineato.

Altra interferenza con la [1] sarebbe da attendere per la dissociazione del vapor d'acqua. Vicerersa un rapidlo calcolo orientativo, elfettuato per condizioni di bassa pressione e alta temperatura, che sono le più farorevoli alla dissociazione del vapor d'acqua, ha portato a stabilire che tale influenza, a calusa del valore della costante di equilibrio di quest'ultima reazione, ì tanto modesta da essere trascurabile nei limiti di approssimazione assunti.

In conclusione possiamo dire che il quadro delineato circa la stabilità del metano in precedenza mediante lo schema

$$
\mathrm{CO}_{2}+4 \mathrm{H}_{2} \longleftrightarrow \mathrm{CH}_{1}+2 \mathrm{H}_{2} \mathrm{O}_{\text {vapore }}
$$

resta sostanzialmente inalterato anche prendendo in considerazione la reazione del gas d'acqua e la dissociazione termica del valpor d'acqual.

Ne, a'altro canto, nuovi elementi qualitativi sono da attendersi considemando come partecipante all'equilibrio generable anche la dissociazione termica del metano.

\section{б. - Deduzioni.}

Vediamo ora di trame qualehe conclusione da quanto precedentemente esposto.

Tabella 2

Sistema di reazioni $\mathrm{CO}_{2}++\mathrm{H}_{2} \underset{\mathrm{C}}{\longrightarrow} \mathrm{CH}_{4}+2 \mathrm{H}_{9} \mathrm{O} \quad \mathrm{CO}_{2}+\mathrm{H}_{2} \underset{\mathrm{C}}{\longleftrightarrow} \mathrm{OO}+\mathrm{H}_{2} \mathrm{O}$

Frazioni molari di $\mathrm{CH}_{4}$ in equilibrio per varie condizioni di pressione e temperatura. La composizione iniziale del sistema è: $n_{H_{9} \mathrm{O}}=0,89 ; n_{\mathrm{CO}_{2}}=0,10: u_{n_{z}}-0,01$

\begin{tabular}{ccc}
\hline$P($ Atmosfere $)$ & $T=1200^{\circ} \mathrm{K}$ & $T=1600^{\circ} \mathrm{K}$ \\
\hline 100 & $\mathrm{tr}$ & $\mathrm{tr}$. \\
500 & $9 \cdot 10^{-7}$ & $\mathrm{tr}$. \\
800 & $2 \cdot 10^{-6}$ & $\mathrm{tr}$. \\
1000 & $+\cdot 10^{-6}$ & $1 \cdot 10^{-7}$ \\
2000 & $2 \cdot 10^{-5}$ & $4 \cdot 10^{-7}$ \\
3000 & $3 \cdot 10^{-5}$ & $9 \cdot 10^{-7}$ \\
\hline
\end{tabular}


Per quanto riguarda la possibilità di equilibrio del metano si dovebbe dedure che essa ì esclusa in ambiente caratterizzato da elevata temperatura (intorno ai $1000{ }^{\circ} \mathrm{C}$ ) e bassa pressione (prossina all'atmosferica).

Detto ambiente chimico-fisico pare identificabile, dal punto di rista rulcanologico, con l'ambiente calatteristico del vulcanesimo di piattaforma.

Detto vulcanesimo i, come noto, caratterizzato da un'attività prevalentemente effusiva, per cui i fluidi possono seguire, al graduale variare della pressione, ma trasformazione chimico-fisica restando in equilibrio con l'ambiente. Dalla lara, una volta pervenuta a giorno, puó liberarsi un miscuglio gassoso di composizione corrispondente alle condizioni di pressione e temperatura determinate dalla lava fusa e dalla pressione atmosferica.

In questo quadro si inserisce l'assenza di metano riscontrata da L. Tay e E. S. Shepherd ( $\left.{ }^{7}\right)$ nelle classiche ricerche dei gas emessi dal Lílauea.

Sensibilmente diverso da quello ora considerato è l'ambiente chimico-fisico caratteristico dei vulcani di materiali incoerenti avendo questi, come è noto, un'attività caratterizzata da fenomeni prevalentemente esplosivi.

Poichè la fase esplosiva è precerluta da una fase preparatoria, durante la quale la pressione ragraiunge valori elevati, se in questa fase le condizioni sono favorevoli alla stabilità del metano, la velocità del fenomeno esplosivo, il rapido abbassamento di temperatura causato dall'espansione dei gas, e la conseguente diminuzione delle velocità di reazione, giustificherebbero la presenza di metano nei prodotti gassosi che si sprigionano nell'atmosfera.

In altri termini, in roncomitanza ron i fenomeni esplosivi, sarebbero da attendersi reri e propri fenomeni di "tempera" del sistema gassoso per cui esso conserverebbe una composizione prossima a quella relativa alla fase di alta pressione precelente l'esplosione.

Esaminiamo ora l'ambiente del plutonismo tipico; esso è caratterizzato da una temperatura sensibilmente inferiore e da pressioni superiori rispetto ai valori caratteristici dell'ambiente vulcanico di piattaforma.
Circa la temperatura sono da attendersi, secondo Rittmann, valori massimi dell'ordine degli $800^{\circ} \mathrm{C}$. La pressione è in relazione alla profondità: per profondità dell'ordine della diecina di migliaia di metri la pressione sarà dell'ordine di grandezza di qualche migliaio rli atmosfere.

Secondo quanto precedentemente esposto l'ambiente plutonico sarebbe compatibile con la stabilità del metano.

Infine, per quanto riguarda l'ambiente subvalcanico e ipoabissico, non possiamo stabilire un quadro semplice; ciò perchè da caso a caso possono variare sensibilmente gli effetti combinati delle diverse pressioni e temperature.

\section{Conclusioni.}

Quanto precede mette in evidenza come gli idrocarburi contenuti dai fluidi termali non possano riguardarsi, come tali, prova della non jurenilità, in senso lato, dei fluidi stessi.

I infatti lecito ritenere che solo in taluni casi tali irlrocarburi possano essere interpretati con sicurezza come secondari, nel senso di non originali, del "magma" e in questa ipotesi essi possono aver origine per una duplice via: una volta che fluidi termali abbiano abbandonato le masse ignee che li hanno generati, per reazioni chimiche in seno ai fluidi stessi e senzal apporto di materia da parte dei termeni attraversati; oppure per scambio di materia con i terreni, per reazioni chimiche o fenomeni puramente fisici o mediante amberlue i meccanismi.

Inoltre è da ritenere che, per particolari condizioni di pressione o temperatura, anche in seno alle masse ignee possono permanere indissociate quantità di metano analiticamente rilevabili, e, in questo caso, l'idrocarburo sarebbe juvenile, ovvero originario della massa magmatica.

Roma (S. Pietro in Vincoli), Centro di Studio per la Geologia T'ecnica del O.N.R. presso ?'Istituto di Geologia Applicata e di Giacimenti Minerari della Facoltà d'Ingegneria, Università di Roma, Agosto 1958. 


\section{RIASSUNTO}

Da alcuni anni è stato posto il quesito se la presenza di idrocarburi in seno a fluidi esalativi $e$ idrotermali sia da interpretare come indizio di non "juvenilità " dei fluidi stessi.

Allo scopo di raccogliere qualche idea in proposito, lo scricente ha studiato per ria teorica alcuni equilibri chimici, cercando in un primo tempo di vedere quali condizioni di pressione e temperatura siano compatibili con la stabilita del metano, inquadrando, quindi, $i$ risultati dello studio chimico-fisico nei vari ambienti "ignei" (magmatici in senso lato e classico).

Le conclusioni a detta analisi sarebbero che gli idrocarburi riscontrati in rari fluidi termali naturali non possono considerarsi prova di non juvenilità. Ciò perchè in ambienti di alte pressioni $e$ di temperature non molto elevate, quali quelle del plutonismo tipico, il metano non è completamente dissociato.

La completa dissociazione del metano, viceversa, sembrerebbe verificarsi nell'ambiente del vulcanesimo di piattaforma. Questo è caratterizzato, come è noto, da un'attività di tipo prevalentemente effusico; il tranquillo decorso di essa dovebbe permettere ai lluidi termali di mantenersi in equilibrio termodinamico con l'ambiente, il quale, essendo caratterizzato da elevatissime temperature $e$ pressioni decrescenti fino all'atmosferica, via via che il magma effonde, sarebbe incompatibile con la stabilitì del metuao.

\section{ABSTRACT}

In the last fer years the question has been raised if presence of hydrocarbons in cirhalative and hydrothermal fluids can or cam not be considered as a suggestion of non "jucenile" origin of these fluids. With the aim of piching' $u$ p some ideas about this problem the Autor studied theorically some chemical equilibria, trying at first to see what pressure and temperature conditions are compatible with the stability of methane. Afterwards, the result of this physico-chemical study were compared with the lnown conditions of the different igneous milieus (magmatic in the wide and classical meaning).

The conditions of such analysis rould be that hydrocarbons foumd in various fluids do not exclude jurenilism: this because at high pressures and not rery high temperatures, tilie these reigning in the typical plutonic conditions, methane is not completly dissociated.

Complete dissociation of methane, on the contrary, seems to talie place in the conditions of continental platean vulcanism, This one is chavacterised, as known, by an activity of a mostly effusice type; the activity proceeds quietly and should allow the thermal fluids: to remain in thermadynamical equilibrium with the medium.

During the effusion, indeed, temperatures remain rery high, while pressures decrease dou'n to the atmospheric ralue, as magma is flowing; these conditions would therefore be incompatible with the stability of methene.

\section{BHIBIIOCRAPIA}

(1) PEsтa, F., Camalleristiche e genesi delle manifestazioni esalalio-idrolermali nalurali, Atti XIV rimione della SIPS, Sapoli, I954. - Vedi anche Pexta. F.. e DI Nor, A.. S'ul signifiealo genelico degli idroearburi nelle manifeslazioni esalative ed idrotermali. "Chimica e Inge. gneria ", 1. Roma. 1956. A queste rlue mote si rimanda per la terminologia, yui, aloperata.

(2) Ellas, A. .T., Chemian equilibrium in magmatic gatses. "American Jomrual of Science" $" 255$, 6. 4I6-43 I (I957).

(3) Ritwanx. A. Tuleani: Allivili e genesi. Erl. Politecuica S. A., Napoli, 1944.

(4) Wansstom, E. E., Introduction to theo. retieal igneous petrology Wiley a Soms Inc., New Tork, 1950 .

(5) ErLofe, (i.. The reaclion of pure hyelrocarbons. "Reinliold Pub. Corp." New Jork, 1937.

(6) Eganert. I., Trallalo di phimica fisica ed clellochimica. 6a Ediz. Italiama, Ed. Hoepli. Milano, I949.

(7) C'LARке, J'. W.. The dala of Geochemislry. "I. S. Cieolopical survey Bull.", 770 , 1924. 